\title{
A Multiparameter Chaos Control Method Applied to Maps
}

\author{
Aline Souza de Paula and Marcelo Amorim Savi \\ Universidade Federal do Rio de Janeiro \\ COPPE - Department of Mechanical Engineering \\ 21.941.972 - Rio de Janeiro - RJ, Brazil, P.O. Box 68.503 \\ E-Mail: alinesp@ufrj.br,savi@mecanica.ufrj.br
}

(Received on 25 February, 2008)

\begin{abstract}
Chaos is a kind of nonlinear system response that has a dense set of unstable periodic orbits (UPOs) embedded in a chaotic attractor. The idea of the chaos control is to explore the UPO stabilization obtaining dynamical systems that may quickly react to some new situation, changing conditions and their response. The OGY (Ott-Grebogi-Yorke) method achieves system stabilization by using small perturbations promoted in the neighborhood of the desired orbit when the trajectory crosses a specific surface, such as a Poincaré section. This contribution proposes a multiparameter (MP) method based on OGY approach in order to control chaotic behavior using different control parameters. As an application of the proposed multiparameter general formulation it is presented an uncoupled approach where the control parameters do not influence the system dynamics when they are not active. This method is applied to control chaos in maps using two control parameters. The two-dimensional Hénon and Ikeda maps are of concern. Results show that the proposed procedure can be a good alternative for chaos control since it provides a more effective UPO stabilization than the classical singleparameter OGY approach.
\end{abstract}

Keywords: Chaos; Control; Nonlinear dynamics; Hénon map; Ikeda map; OGY method

\section{INTRODUCTION}

Chaos control explores the intrinsic richness of the chaotic behavior by stabilizing unstable periodic orbits (UPOs) embedded in a chaotic attractor. This control method is based on some chaotic behavior properties as the existence of a dense set of UPOs embedded in a chaotic attractor; the ergodicity property; and the sensitive dependence to initial condition. Therefore, chaos is related to flexibility since, when controlled, may allows the system to quickly change from one kind of response to another.

Chaos control methods may be classified as discrete or continuous techniques. The modern study of chaos control starts with the pioneer method proposed by Ott et al. [1], nowadays known as the OGY method as a tribute of their authors (Ott-Grebogi-Yorke). This is a discrete technique that considers small perturbations of a single-parameter applied in the neighborhood of the desired orbit [2,3]. On the other hand, a different approach explores the delayed feedback control proposed by Pyragas [4] that states the stabilization of chaotic systems by a feedback perturbation proportional to the difference between the present and a delayed state of the system. The original limitations of the OGY method are overcome in different references and, among others, one can cite: control of high periodic and high unstable UPO [5-9], control using time delay coordinates [10-13], control using different control parameters $[14,15]$.

Chaos control techniques are being used in different applications in order to stabilize UPOs. The first experimental verification of the OGY approach is applied to magneto-elastic beams [16]. Andrievskii \& Fradkov [17] present an overview of chaos control applications in various scientific fields. Mechanical systems are included in this discussion presenting control of pendulums, beams, plates, vibroformers, microcantilevers, cranes and vessels.

This contribution considers a multiparameter chaos control method built upon the OGY approach. The idea is to use different control parameters in order to perform the UPO stabilization and, because of that, the map that establishes the relation between the system responses in two subsequent control stations depends on all control parameters. The proposed method assumes that only one control parameter is perturbed in each control station, defining active (is perturbed in a control station) and passive (is not perturbed in a control station) parameters. As an application of the general formulation, an uncoupled approach is proposed where control parameters return to their reference value when they become passive and therefore, they are not influencing the system dynamics. This method is applied to control chaos in maps considering two control parameters. Specifically, Hénon and Ikeda maps are of concern. Hénon map is a simple two-dimensional map with quadratic non-linearity that gave a first example of the strange attractor with a fractal structure [18]. As suggested by Hénon [19], this two-dimensional map is a simple version of the original problem that describes fluid flow in three-dimensional space, exhibiting the same properties. Ikeda map is also a two-dimensional map that originally represents a string of light pulses impinging on a partially transmitting mirror [20]. De Paula \& Savi [14] use similar idea of the multiparameter method in order to control chaos in a nonlinear pendulum using a semi-continuous method [21]. Lenz \& Obradovic [22] also perform a multiparameter control of Hénon and Ikeda maps employing the locally linearized system representation.

All system dynamics may be treated from time series analysis. Here, the close-return (CR) method [23] is employed to indentify the UPOs embedded in the attractor. Afterwards, the local dynamics expressed by the Jacobian matrix and the sensitivity matrix of the transition maps in a neighborhood of the control points are determined using the least-square fit method $[6,8,13,23,24]$. Moreover, the singular value decomposition (SVD) technique is employed for determining the stable and unstable directions near the control point. Results show that 
the uncoupled multiparameter approach can be a good alternative for chaos control since it provides a more effective UPO stabilization when compared to those obtained from the classical single-parameter OGY approach.

\section{MULTIPARAMETER CHAOS CONTROL METHOD}

Chaos control method is a two stage technique where the first step is the learning stage and the second one is the control stage. The learning stage identifies the unstable periodic orbits and evaluates some system characteristics. The control stage, on the other hand, controls the desirable UPOs promoting their stabilization.

After the learning stage, one can proceed to the next stage where a control process needs to be used to stabilize a desired orbit. The OGY approach is an alternative to promote this UPO stabilization. Its description considers a discrete system equivalent to a parameter dependent map associated with a general surface, usually a Poincaré section. Let $F$ be the mapping of the system behavior from one Poincaré section $\Sigma_{n}$ to the next one $\Sigma_{n+1}$ and $\xi^{n}$ be the intersection of the system trajectory with the Poincare section $\Sigma_{n}$. Therefore, it is possible to write:

$$
\xi^{n+1}=F\left(\xi^{n}, p\right)
$$

where $p \in \Re$ is an accessible parameter for control. The control idea is to monitor the system dynamics in these control stations until the neighborhood of a desirable fixed point is reached. After that, a proper small change in the parameter $p$ causes the next state $\xi^{n+1}$ to fall into the stable direction of the fixed point. In order to find the proper variation in the control parameter, perturbation $\delta p$, it is considered a linearized version of the dynamical system near this control point. The linearization has a homeomorphism with the nonlinear problem that is assured by the Hartman-Grobman theorem [24].

The proposed multiparameter (MP) chaos control method formulation, introduced in reference [14] as a semicontinuous method, is now presented in order to provide a better reading. The multiparameter control considers $N_{p}$ different control parameters, $p_{i}\left(i=1, \ldots, N_{p}\right)$. By considering a specific control station, only one of those control parameters is perturbed. Under this assumption, the map $F$, that establishes the relation of the system behavior between the control stations $\Sigma_{n}$ and $\Sigma_{n+1}$, depends on all control parameters. In general, although only one parameter is perturbed in each section, it is assumed the influence of all control parameters based on their positions in station, $\Sigma_{n}$. On this basis,

$$
\xi^{n+1}=F\left(\xi^{n}, P^{n}\right)
$$

where $P^{n}$ is a vector that contains all control parameter positions. By using a first order Taylor expansion, one obtains the linear behavior of the map $F$ in the neighborhood of the control point $\xi_{C}^{n}$, that consist in the intersection of the desired
UPO with control station $\Sigma_{n}$, and around the control parameters reference positions, $P_{0}$, as defined by Eq. (3).

$$
\left.\delta \xi^{n+1} \cong D_{\xi^{n}} F\left(\xi^{n}, P^{n}\right)\right|_{\xi^{n}=\xi_{C}^{n}, P^{n}=P_{0}} \delta \xi^{n}+
$$

$$
\left.D_{P^{n}} F\left(\xi^{n}, P^{n}\right)\right|_{\xi^{n}=\xi_{C}^{n}, P^{n}=P_{0}} \delta P^{n}
$$

This equation may be rewritten as follows

$$
\delta \xi^{n+1} \cong J^{n} \delta \xi^{n}+W^{n} \delta P^{n}
$$

where $\delta \xi^{n+1}=\xi^{n+1}-\xi_{C}^{n+1}, \quad \delta \xi^{n}=\xi^{n}-\xi_{C}^{n}, \quad \delta P^{n}=$ $P^{n}-P_{0}$ is related to the parameter perturbations, $W^{n}=$ $\left.D_{P^{n}} F\left(\xi^{n}, P^{n}\right)\right|_{\xi^{n}=\xi_{C}^{n}, P^{n}=P_{0}}$ is the sensitivity matrix in which each column is related to a control parameter and $J^{n}=$ $\left.D_{\xi^{n}} F\left(\xi^{n}, P^{n}\right)\right|_{\xi^{n}=\xi_{C}^{n}, P^{n}=P_{0}}$ is the Jacobian matrix. Moreover, from Jacobian eigenvalues $\left(e_{u}, e_{s}\right)$ it is possible to define a contravariant basis vector $\left(f_{u}, f_{s}\right)$ given by $f_{s} \cdot e_{s}=f_{u} \cdot e_{u}=1$, $f_{s} \cdot e_{u}=f_{u} \cdot e_{s}=0$. Therefore, the Jacobian can be written as:

$$
J^{n}=\lambda_{u} e_{u} f_{u}+\lambda_{s} e_{s} f_{s}
$$

By assuming that only one parameter is perturbed in each control station, it is possible to define active parameters, represented by subscript $a, \delta P_{a}^{n}$ (is perturbed in station $\Sigma_{n}$ ), and passive parameters, represent subscript $p, \delta P_{p}^{n}$ (is not perturbed in station $\left.\Sigma_{n}\right)$. Hence,

$$
\delta \xi^{n+1} \cong J^{n} \delta \xi^{n}+W_{a}^{n} \delta P_{a}^{n}+W_{p}^{n} \delta P_{p}^{n}
$$

where $W_{a}^{n}$ is the sensitivity matrix column related to the active parameter in control station $\Sigma_{n}$ and $W_{p}^{n}$ consists on the sensitivity matrix columns related to the passive parameters in the same control station.

The foregoing formulation may be considered as a general multiparameter control method where all parameters perturb the system dynamics. A particular case of this general procedure has uncoupled control parameters meaning that each parameter returns to its reference value when it becomes passive. Under this assumption, passive influence vanishes:

$$
W_{p}^{n} \delta P_{p}^{n}=0
$$

Therefore, the map $F$ is just a function of the active parameter, $\xi^{n+1}=F\left(\xi^{n}, P_{a}^{n}\right)$, and the linear behavior of the map $F$ in the neighborhood of the control point $\xi_{C}^{n}$ and around the control parameter reference positions, $P_{0}$, is now defined by:

$$
\delta \xi^{n+1} \cong J^{n} \delta \xi^{n}+W_{a}^{n} \delta P_{a}^{n}
$$

In order to pick $\delta P_{a}^{n}$ in such a way that $\delta \xi^{n+1}$ falls on the stable direction of the desired fixed point, it is considered the following restriction: 


$$
f_{u} \cdot \xi^{n+1}=0
$$

Thus, for sufficiently small $\delta \xi^{n}$ and using the Jacobian defined in (5), one obtains:

$$
\delta P_{a}^{n}=-\lambda_{u} \frac{f_{u} \cdot \delta \xi^{n}}{f_{u} \cdot W_{a}^{n}}
$$

where $\delta P_{a}^{n}$ corresponds to the perturbation that must be applied by the active parameter in control station $\Sigma_{n}$ in order to stabilize the system trajectory.

\section{CONTROLLING MAPS}

Numerical simulations considering maps are carried out in order to evaluate the capability of the proposed uncoupled multiparameter chaos control method to stabilize desirable UPOs. System characteristics are evaluated from time series generated by map iterations. Specifically, the twodimensional Hénon and Ikeda maps are of concern. Since both cases are related to discrete-time nonlinear system, the system dynamics automatically furnishes the Poincaré map construction. Therefore, the control action is directly applied in map iterations.

In the first stage of the control strategy, UPOs embedded in the chaotic attractor are identified. The close return method [23] is employed with this aim. The UPO identification is done by considering a number of map iterations and a prescribed tolerance as follows:

$$
\left\|X_{n+P}-X_{n}\right\| \leq \text { tolerance }
$$

where $\left\|X_{n+P}-X_{n}\right\|$ is a function that returns the largest singular value of $\left(X_{n+P}-X_{n}\right)$ and the subscript $P$ indicates the periodicity of the identified UPO.

After the UPO identification, the local dynamics expressed by the Jacobian matrix and the sensitivity matrix of the transition maps in a neighborhood of the control points are determined using the least-square fit method [6,8,13,23,24]. The sensitivity matrices are evaluated allowing trajectories to come close to a control point and then one perturbs the parameters by proportional value of the maximum permissible. After that, the singular value decomposition (SVD) technique is employed for determining the stable and unstable directions near the next control point. At this point, the first stage of the control strategy is completed. In control stage, the perturbation is calculated and, when it is greater than the maximum value, it is assumed a perturbation equal to the maximum value.

The uncoupled multiparameter approach is treated by evaluating a control rule considering a sequence of four different UPOs for each analyzed system. Moreover, in order to establish a comparison between the uncoupled multiparameter and the single-parameter method, the same control rule is applied to both approaches. Two different control parameters

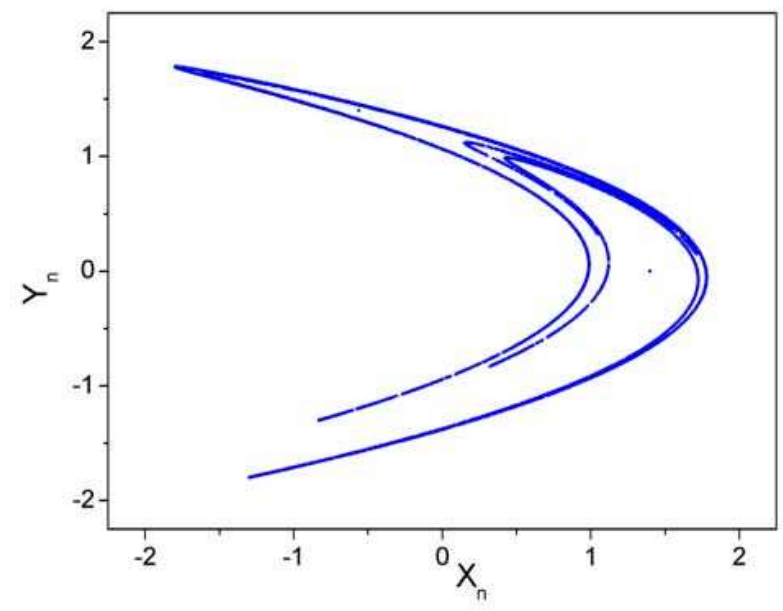

FIG. 1: Chaotic attractor for the Hénon map with parameters values $\alpha=1.4$ and $\beta=0.3$.

are analyzed in the single-parameter approach considering the isolated perturbation performed by each parameter employed during multiparameter method.

As an application of the proposed formulation, it is analyzed the uncoupled multiparameter chaos control method applied to the Hénon and to the Ikeda maps using two control parameters. Under this assumption, each system iteration represents the desired Poincaré map. Moreover, since in each control station only one control parameter is perturbed, it is considered that they are perturbed alternating each other. Finally, the evaluation of the control parameter is given by Eq.(10), considering the column of the sensitivity matrix associated with the active control parameter.

\subsection{Controlling Hénon Map}

The Hénon map is a simple two-dimensional map with quadratic non-linearity. Mathematically, it can be expressed as follows [19]:

$$
\begin{aligned}
X_{n+1} & =(\alpha+\delta \alpha)+(\beta+\delta \beta) Y_{n}-X_{n}^{2} \\
Y_{n+1} & =X_{n}
\end{aligned}
$$

where $\delta \alpha$ is the first control parameter while $\delta \beta$ is the second control parameter.

By considering a chaotic strange attractor of the Hénon map (Fig. 1) for parameter values $\beta=0.3$ and $\alpha=1.4$, it is applied the multiparameter control approach, with two control parameters, as well as the single-parameter approach.

The UPO identification is done by considering 10,000 map iterations and a prescribed tolerance of 0.01 . Under this assumption, 32 UPOs are identified during the learning stage up to periodicity 15 . It is defined a control rule that considers the following UPO sequence: a period-1 orbit during the first 500 iterations, a period- 15 from iteration 500 to 1000 , a period- 4 from 1000 to 1500 and, finally a period-7, from period 1500 to 2000 . 

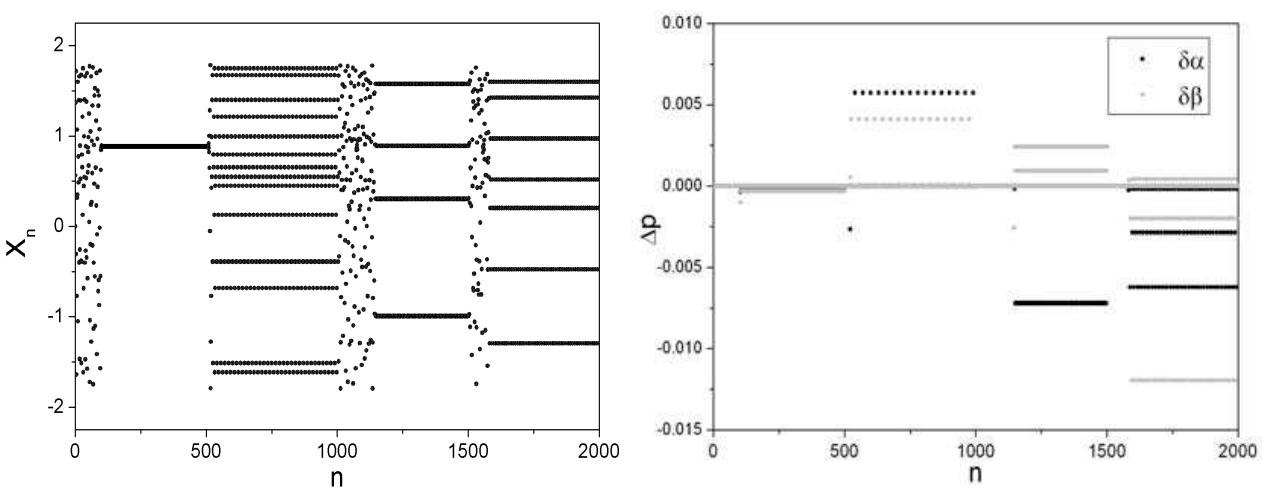

FIG. 2: Hénon map stabilization using uncoupled multiparameter approach: (a) Displacement; (b) Control parameters perturbations.
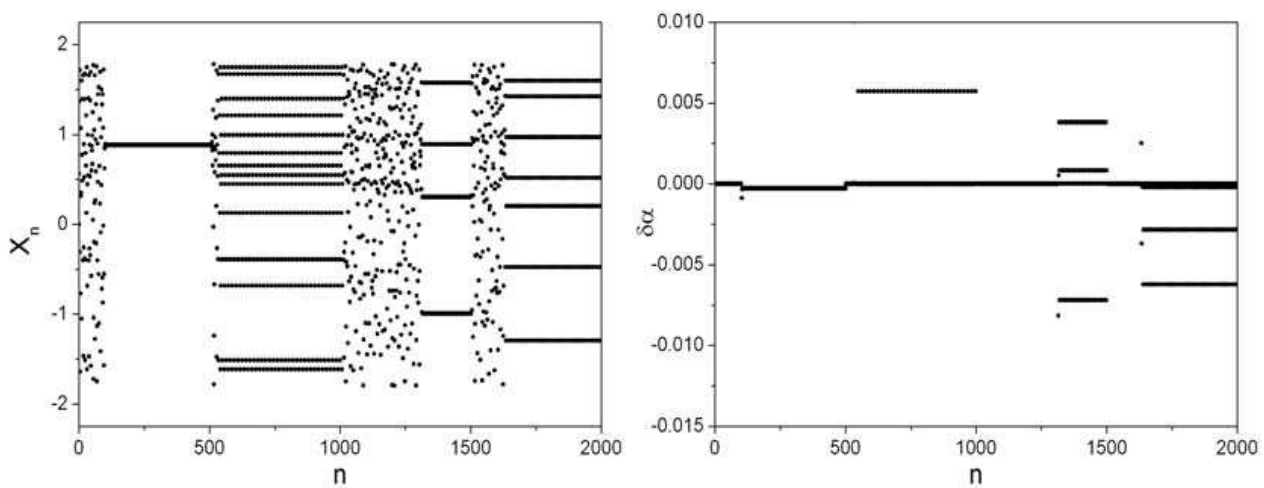

FIG. 3: Hénon map stabilization using single-parameter $\delta \alpha$ : Displacement; (b) Control parameters perturbations.
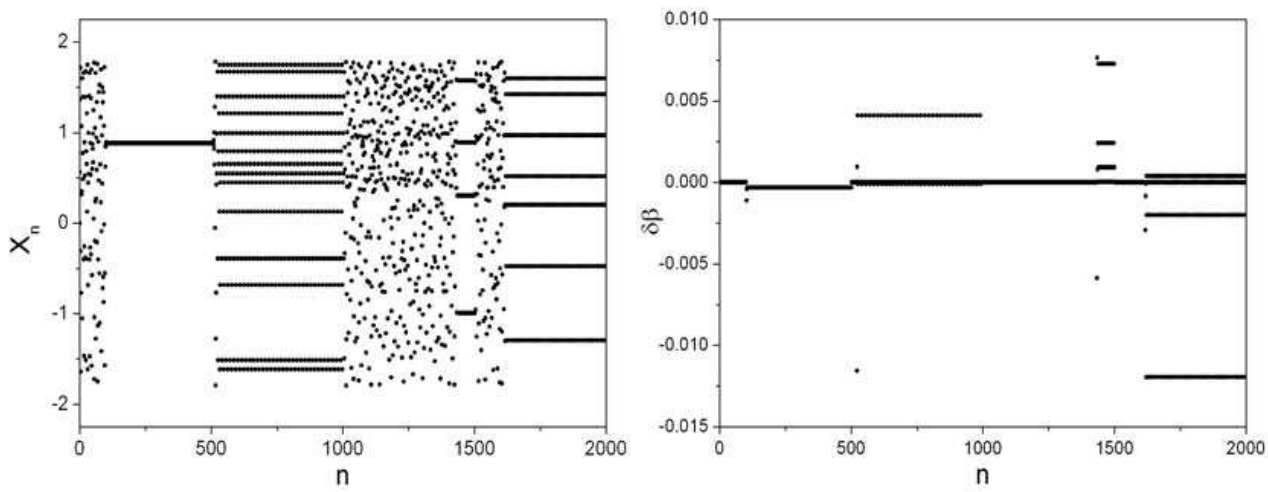

FIG. 4: Hénon map stabilization using single-parameter $\delta \beta$ : (a) Displacement; (b) Control parameters perturbations.

In chaos control evaluation it is considered $\delta \alpha_{\max }=$ $\delta \beta_{\max }=0.09$ as maximum parameter perturbations. When the calculated perturbation is greater than these values it is assumed a perturbation equal to the maximum value. The reference positions considered for both parameters are $\delta \alpha_{0}=$ $\delta \beta_{0}=0$.

Figure 2 presents results for the cited control rule employing the uncoupled multiparameter control approach. On the other hand, results for single-parameter approach, considering the isolated perturbation performed by each control parame- ter, are shown in Fig. 3 (where $\delta \alpha$ is the control parameter) and in Fig. 4 (where $\delta \beta$ is the control parameter). All these Figures present the evaluated control perturbation. In Fig. 2, both parameter perturbations are presented together, however, it is important to highlight that when one of the control parameters is perturbed the other is always at its reference position. These results show that both multiparameter and singleparameter approaches are effective to stabilize all UPOs of the cited control rule. 


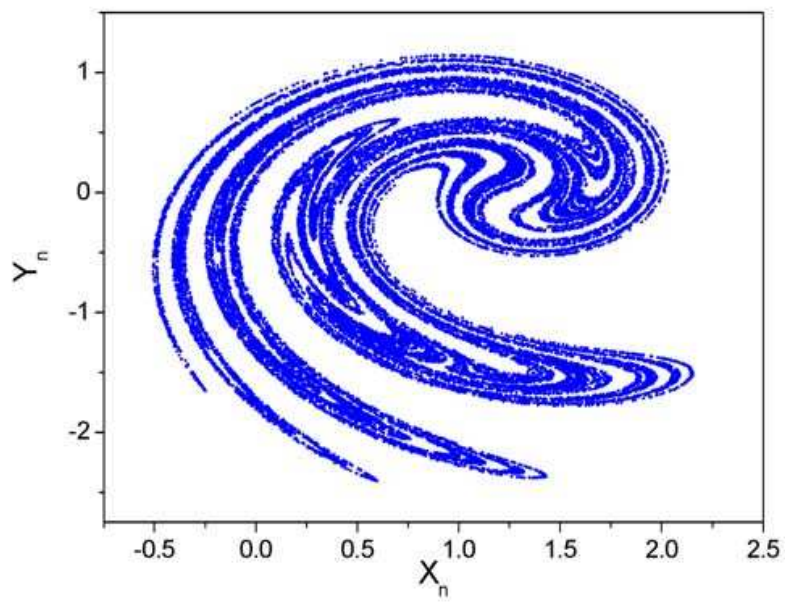

FIG. 5: Chaotic attractor for the Ikeda map with parameters values $\beta=0, \gamma=7, \mu=0.9$ and $\alpha=1.25$.
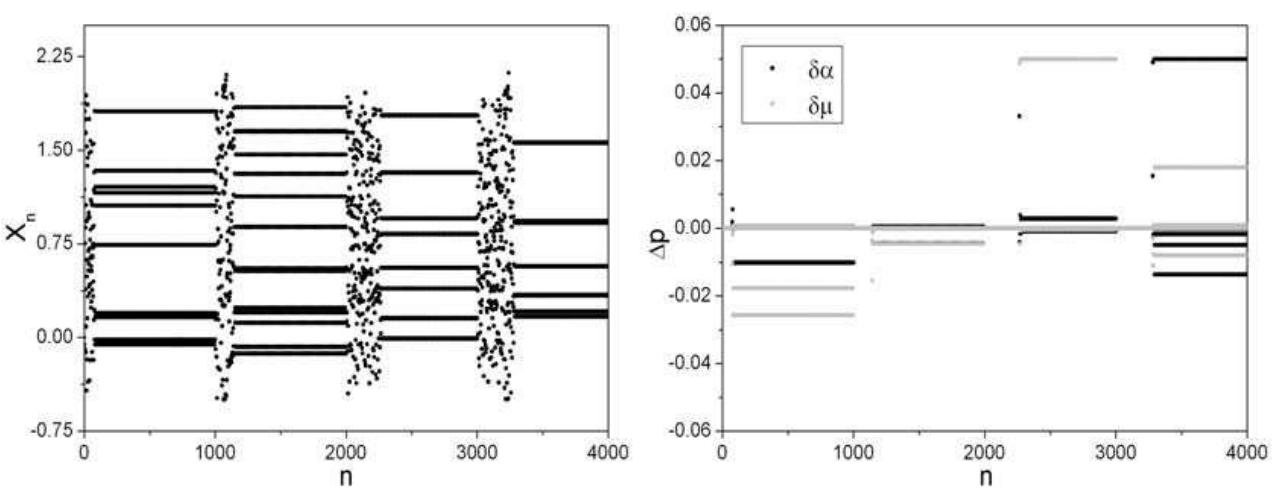

FIG. 6: Ikeda map stabilization using uncoupled multiparameter approach: Displacement; (b) Control parameters perturbations.

\subsection{Controlling Ikeda Map}

The Ikeda map is a two-dimensional map that represents a string of light pulses entering at the partially transmitting mirror [20], being mathematically expressed as:

$$
\begin{aligned}
X_{n+1} & =(\alpha+\delta \alpha)+(\mu+\delta \mu)\left(X_{n} \cos \left[t_{n}\right]-Y_{n} \sin \left[t_{n}\right]\right) \\
Y_{n+1} & =(\mu+\delta \mu)\left(X_{n} \sin \left[t_{n}\right]+Y_{n} \cos \left[t_{n}\right]\right)
\end{aligned}
$$

where $t_{n}=\beta-\frac{\gamma}{1+X_{n}^{2}+Y_{n}^{2}}, \delta \alpha$ is the first control parameter while $\delta \mu$ is the second control parameter.

In chaos control evaluation it is considered $\delta \alpha_{\max }=$ $\delta \mu_{\max }=0.05$ as maximum parameter perturbations. Once again, when the calculated perturbation is greater than these values it is assumed a perturbation equal to the maximum value. The reference positions considered for both parameters are $\delta \alpha_{0}=\delta \mu_{0}=0$.

The uncoupled multiparameter approach, considering two control parameters, as well as the single-parameter OGY method, is now applied to the chaotic strange attractor of the Ikeda map for the parameter values $\beta=0, \gamma=7, \mu=0.9$ and $\alpha$ $=1.25$ (Fig. 5) [25].
The UPO identification is now focused on by considering 30,000 map iterations and a tolerance of 0.005 , assuming the same procedure of the preceding section. Under this assumption, 8 UPOs are identified during the learning stage up to periodicity 15 . The control rule considers the following sequence: a period-10 orbit during the first 500 iterations, a period-14 from iteration 500 to 1000 , a period- 8 from 1000 to 1500 and, finally a period-5, from period 1500 to 2000 .

Control rule sequence is now analyzed during the control stage. Fig. 6 presents results of the control rule stabilization employing the uncoupled multiparameter approach, while results for single-parameter approach, considering the isolated perturbation performed by each control parameter, are shown in Fig. 7 (where $\delta \alpha$ is the control parameter) and in Figure 8 (where $\delta \mu$ is the control parameter). Once again, these Figures present the evaluated control perturbation. Both parameter perturbations are presented together in Fig. 6 where it might be highlighted that when one of the control parameters is perturbed the other is always at its reference position. This result show that the uncoupled multiparameter control approach successfully stabilize all UPOs of the control rule. By observing the single-parameter results it must be noticed that the UPO stabilization is not performed properly. Fig. 7 shows that the 

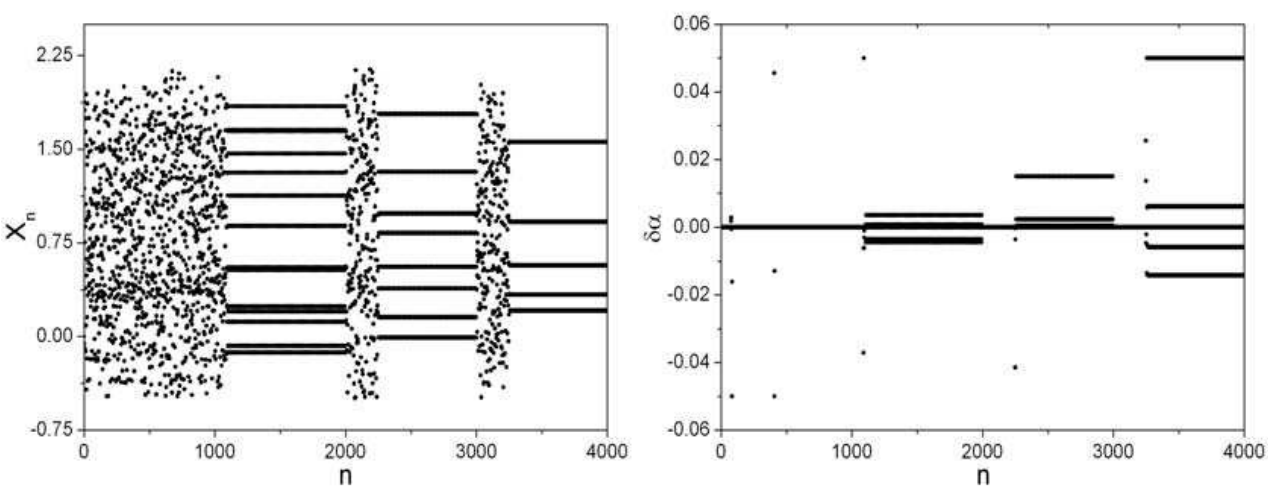

FIG. 7: Ikeda map stabilization using single-parameter $\delta \alpha$ : (a) Displacement; (b) Control parameters perturbations.
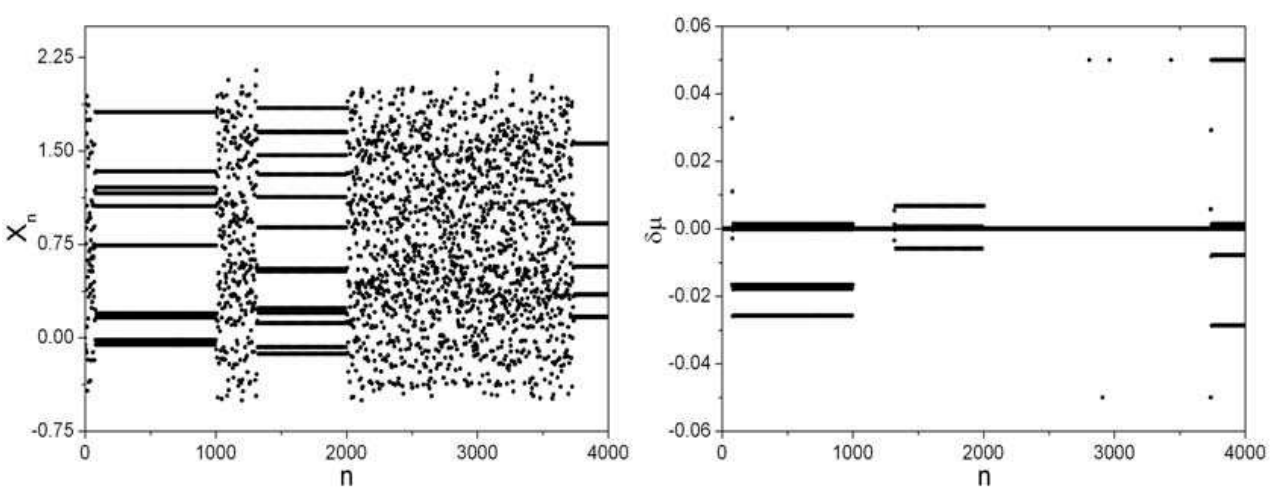

FIG. 8: Ikeda map stabilization using single-parameter $\delta \mu$ : Displacement; (b) Control parameters perturbations.

single-parameter method is not able to stabilize the first UPO when the first parameter $(\delta \alpha)$ is employed as control parameter. On the other hand, the OGY single-parameter method is not able to stabilize the third UPO of the control rule when the second parameter $(\delta \mu)$ is employed (Fig. 8). These results are showing that the multiparameter approach may be more effective in order to perform UPO stabilization.

\section{CONCLUSIONS}

This contribution presents a multiparameter chaos control method built upon the OGY technique. The procedure assumes that only one control parameter is perturbed in each control station, defining active and passive control parameters. As an application of the proposed multiparameter general formulation, it is considered an uncoupled approach where control parameters return to their reference positions when they are not active. The uncoupled multiparameter method is analyzed by evaluating chaos control in Hénon and Ikeda two- dimensional maps with two control parameters. Control rules are defined from a sequence of four different UPOs for each map. Results show that the uncoupled multiparameter procedure successfully stabilizes all orbits controlled by the singleparameter approach. On the other hand, there are situations where the single-parameter approach fails. Therefore, one can conclude that the uncoupled multiparameter procedure tends to be more effective in order to stabilize unstable periodic orbits embedded in the chaotic attractor when compared with the stabilization performed by the single-parameter approach. The application of the general coupled approach is associated with a non-trivial work on defining proper control parameters but may present successful results as shown in De Paula \& Savi [14].

\section{Acknowledgements}

The authors acknowledge the support of Brazilian Research Councils CNPq and FAPERJ.
[1] E. Ott, C. Grebogi, and J. A. Yorke, Phys. Rev. Lett. 64, 1196 (1990).

[2] C. Grebogi and Y. -C. Lai, Syst. Control Lett. 31, 307 (1997).
[3] T. Shinbrot, C. Grebogi, E. Ott, and J. A. Yorke, Nature 363, 411 (1993).

[4] K. Pyragas, Phys. Lett. A 170, 421 (1992). 
[5] B. Hübinger, R. Doerner, W. Martienssen, M. Herdering, R. Pitka, and U. Dressler, Phys. Rev. E 50, 932 (1994).

[6] M. Otani and A. J. Jones, Guiding Chaotic Orbits, Research Report - Imperial College of Science Technology and Medicine (London, 1997).

[7] T. Ritz, A. S. Z. Schweinsberg, U. Dressler, R. Doerner, B. Hübinger, and W. Martienssen, Chaos Sol. \& Fract.8, 1559 (1997).

[8] F. H. I. Pereira-Pinto, A. M. Ferreira, and M. A. Savi, Chaos Sol. \& Fract. 22, 653 (2004).

[9] M. A. Savi, F H. I. Pereira-Pinto, and A. M. Ferreira, Shock Vib. 13, 301 (2006)

[10] U. Dressler and G. Nitsche, Phys. Rev. Lett. 68, 1 (1992).

[11] P. So and E. Ott, Phys. Rev. E 51, 2955 (1995).

[12] R. J. de Korte, J. C. Schouten, and C. V. M. van den Bleek, Phys. Rev. E 52, 3358 (1995).

[13] F. H. I. Pereira-Pinto, A. M. Ferreira, and M. A. Savi, Int. J. Bif. and Chaos 15, 4051 (2005).

[14] A. S. De Paula and M. A. Savi, Chaos Sol. \& Fract. doi:10.1016/ j.chaos.2007.09.056 (2007)
[15] E. Barreto and C. Grebogi, Phys. Rev. E 54, 3553 (1995).

[16] W. L. Ditto, S. N. Rauseo, and M. L. Spano, Phys. Rev. Lett. 65, 3211 (1990).

[17] B. R. Andrievskii and A. L. Fradkov, Automation and Remote Control 65, 505 (2004).

[18] M. Sonis, Chaos Sol. \& Fract 7, 2215 (1996).

[19] M. Hénon, Commun. Math. Phys. 50, 69 (1976).

[20] S. Hammel, C. K. R. T. Jones, and J. Maloney, J. Opt. Soc. Am. B 552, (1985).

[21] A. S. De Paula, M. A. Savi, and F. H. I. Pereira-Pinto, J. Sound Vib. 294, 585 (2006).

[22] H. Lenz and D. Obradovic, Int. J. Bifurcation and Chaos, 9, 251 (1999).

[23] D. Auerbach, P. Cvitanovic, J. -P. Eckmann, G. Gunaratne, and I. Procaccia, Phys. Rev. Lett. 58, 2387 (1987).

[24] M. A. Savi, Nonlinear Dynamics and Chaos , Editora E-papers (2006).

[25] P. M. Alsing, A. Gavrielides, and V. Kovanis, IEEE Nonlinear Optics: Materials, Fundamentals, and Applications (1994) 72. 